\title{
Induced Cosmological Constant in Braneworlds with Compact Dimensions
}

\author{
A. A. $\operatorname{Saharian}^{1 *}$, H. G. Sargsyan ${ }^{1}$ \\ ${ }^{1}$ Department of Physics, Yerevan State University, 1 Alex Manoogian Street, 0025 Yerevan, \\ Armenia \\ *E-mail: saharian@ysu.am
}

\begin{abstract}
We investigate the cosmological constant induced by quantum fluctuations of a bulk charged scalar field on a brane in background of locally anti-de Sitter spacetime with toroidally compact spatial dimensions. Along compact dimension quasiperiodicity conditions are imposed with general phases and, in addition, the presence of a constant gauge field is assumed. The latter gives rise to Aharonov-Bohm type effect on the characteristics of the scalar vacuum. The renormalization of the vacuum energy density on the brane is done by making use of the generalized zeta function technique. The behavior of the cosmological constant is studied as a function of the location of the brane, of the length of the compact dimensions and of the magnetic flux enclosed by the compact dimension. In particular, it is shown that the cosmological constant is a periodic function of the magnetic flux with the period equal to the flux quantum.
\end{abstract}

Keywords: cosmological constant - braneworld scenario - anti de Sitter spacetime - Casimir effect.

\section{Introduction}

The braneworld models (for reviews see Brax 2003) and Maarten (2010) provide an interesting alternative to the standard Kaluza-Klein compactification of the extra dimensions. In the corresponding constructions the standard model fields are localized on a hypersurface (brane) in a higherdimensional spacetime. As a consequence of that, the observational restrictions on the size of extra dimensions are much weaker. The introduction of large extra spatial dimensions may provide a solution to the hierarchy problem between the gravitational and electroweak mass scales. The main idea to resolve the large hierarchy is that the small coupling of four dimensional gravity is generated by the large physical volume of extra dimensions. Braneworlds naturally appear in string/M-theory context and provide 
a novel setting for discussing phenomenological and cosmological issues related to extra dimensions. The problem of the cosmological constant has been considered as the most serious mass hierarchy problem in modern particle physics (see, for instance, Martin (2012) and many attempts addressing this fine tuning issue can be found in the literature. The braneworld theories may give some alternative discussion of the cosmological constant.

The investigations of quantum effects in braneworlds are of considerable interest in particle physics and cosmology. An inherent feature is the presence of boundaries and the fields that propagate in the bulk will give Casimir-type contributions to the vacuum expectation values (VEVs) of physical observables (for reviews of the Casimir effect see Milton (2002), Bordag et al. (2009), and Casimir Physic (2011)). In particular, vacuum forces arise acting on the branes which can either stabilize or destabilize the braneworld. The Casimir energy gives a contribution to both the brane and bulk cosmological constants and, hence, has to be taken into account in the self-consistent formulation of the corresponding models. Motivated by these issues, the quantum vacuum effects induced by branes in AdS bulk have received a great deal of attention (see references cited in Bezerra de Mello et al, (2015)). The VEV of the energy-momentum tensor has been investigated in Knapman \& Toms (2004).

In manifolds with boundaries the energy-momentum tensor in addition to the bulk part contains a contribution located on the boundary (see Saharian (2004) for a real scalar field). In Saharian $(2004,2006)$ and Saharian et al. (2018)the VEV of the surface energy-momentum tensor is evaluated for a massive scalar field subject to Robin boundary conditions on branes in AdS bulk. It has been shown that in the Randall-Sundrum models the cosmological constant induced on the visible brane by the hidden brane can serve as a model for dark energy. In the present paper we investigate the VEV of the surface energy-momentum tensor for a charged scalar field in locally AdS background spacetime with a codimension-one brane parallel to the AdS boundary and with an extra compactified dimension (generalized 1-brane Randall-Sundrum model). In addition, the presence of a constant gauge field is assumed. It is shown that the vacuum expectation value of the surface energy-momentum tensor on a brane gives rise to a cosmological constant type contribution from the point of view of an observer living on the brane.

\section{Problem setup}

In Poincaré coordinates, the metric tensor for a $(D+1)$-dimensional AdS spacetime is given by the line element

$$
d s^{2}=g_{i k} d x^{i} d x^{k}=e^{-2 y / a} \eta_{\mu \nu} d x^{\mu} d x^{\nu}-d y^{2},
$$


where $\eta_{\mu \nu}=\operatorname{diag}(1,-1, \ldots,-1)$ is the $D$-dimensional Minkowski metric, $\mu, \nu=0,1, \ldots, D-1$, and the indices $i, k$ run over 0 to $D$. In what follows, we use lowercase Latin letters to denote indices running from 0 to $D$, and lowercase Greek letters to denote indices running from 0 to $D-1$. In addition to the coordinate $y, \quad-\infty<y<+\infty$, we introduce the coordinate $z$, defined as $z=a e^{y / a}, \quad 0<z<\infty$. In terms of the latter the line element is written in a manifestly conformally flat form $d s^{2}=(a / z)^{2}\left(\eta_{\mu \nu} d x^{\mu} d x^{\nu}-d z^{2}\right)$. In terms of the coordinate $z$, the hypersurfaces $z=0$ and $z=\infty$ correspond to the AdS boundary and horizon respectively. The Ricci scalar $R$ and the cosmological constant $\Lambda$ are related to the AdS radius $a$ as $R=D(D+1) a^{-2}$ and $\Lambda=-D(D-1) a^{-2} / 2$.

The local geometry we are going to consider here is that of AdS, given by (1), however the global properties differ. It will be assumed that the coordinate $x^{D-1}$ is compactified on a circle $S^{1}$ with the length $L, 0 \leq x^{D-1} \leq$ $L$. For the remaining coordinates, as usual, we take $-\infty<x^{i}<+\infty$, $i=1, \ldots, D-2$. Note that the proper length of the compact dimension measured by an observer with fixed $y$ is given by $L_{(p)}=a L / z=e^{-y / a} L$. The latter is decreasing with increasing $y$.

We are interested in quantum effects on the vacuum state for a complex scalar field $\varphi(x)$ induced by compactification of the dimension $x^{D-1}$. Assuming the presence of an external gauge field $A_{i}$, the corresponding equation of motion reads

$$
\left(g^{i k} D_{i} D_{k}+m^{2}+\xi R\right) \varphi(x)=0
$$

with $D_{k}=\nabla_{k}+i e A_{k}$ being the gauge extended covariant derivative operator. We assume that along the compact dimension the field obeys the quasiperiodicity condition

$$
\varphi\left(t, x^{1}, \ldots, x^{D-1}+L, y\right)=e^{2 \pi i \alpha} \varphi\left(t, x^{1}, \ldots, x^{D-1}, y\right),
$$

with a constant phase $\alpha$. Here we consider a gauge field configuration with constant $A_{i}$. In this case the corresponding field strengths are zero. However due to the nontrivial spatial topology the field $A_{i}$ leads to physical effects. In the geometry under consideration, in the presence of constant external gauge field the VEVs of the current densities along the compact dimensions are investigated in Bezerra de Mell et al. (2015), where it is shown that Aharonov-Bohm like effects arise due to the non-trivial topology. In the same geometry with the presence of branes the VEV of the current density is investigated in Bellucci et al. $(2015,2016)$.

With constant $A_{i}$, by the gauge transformation $\varphi(x)=e^{-i e \chi(x)} \varphi^{\prime}(x)$, $A_{i}=A_{i}^{\prime}+\partial_{i} \chi(x)$, with $\chi(x)=A_{i} x^{i}$, one passes to a new gauge, where $A_{i}^{\prime}=0$. However, the vector potential does not completely disappear from the problem: it enters into the quasiperiodicity condition for the new field

$$
\varphi^{\prime}\left(t, x^{1}, \ldots, x^{D-1}+L, y\right)=e^{2 \pi i \tilde{\alpha}} \varphi^{\prime}\left(t, x^{1}, \ldots, x^{D-1}, y\right),
$$


where $\tilde{\alpha}=\alpha+e A_{D-1} L /(2 \pi)$. In what follows we will work in the gauge $\left(\varphi^{\prime}(x), A_{i}^{\prime}\right)$ with $A_{i}^{\prime}=0$, omitting the primes for the sake of simplicity. In this gauge, the field equation takes the form (2) with $A_{i}=0$. Note that the shift in the phase induced by the gauge field can be written as $e A_{D-1} L /(2 \pi)=-\Phi / \Phi_{0}$, where $\Phi$ is the gauge field flux enclosed by compact dimension and $\Phi_{0}=2 \pi / e$ is the flux quantum. In addition, we assume the presence of a brane located at $y=y_{0}$. On the brane the field obeys the Robin boundary condition

$$
\left(1+\beta^{(j)} n_{(j)}^{i} \nabla_{i}\right) \varphi(x)=0,
$$

where $\beta^{(j)}$ is a constant and $n_{(j)}^{i}$ is the normal to the brane. In general, the coefficients in the boundary condition can be different for the left and right surfaces of the brane. The superscript $(j)$ differentiates between the right surface, $y=y_{0}+0(j=1)$, and the left surface, $y=y_{0}-0(j=2)$, of the brane.

We are interested in the VEV of the surface energy-momentum tensor $T_{i k}^{(\mathrm{s})}$ for the complex scalar field $\varphi(x)$, localized on the brane. The expression for the latter can be found in a way similar to that used in Saharian (2004) for a real scalar field. On the background of a $(D+1)$-dimensional spacetime region $M$ with timelike boundary $\partial M_{s}$ the surface energy-momentum tensor has the form

$$
T_{i k}^{(\mathrm{s})}=\delta\left(x ; \partial M_{s}\right)\left[\xi \varphi \varphi^{\dagger} K_{i k}-(\xi-1 / 4) h_{i k} n^{l} \nabla_{l}\left(\varphi \varphi^{\dagger}\right)\right],
$$

where $h_{i k}=g_{i k}+n_{i} n_{k}$ is the induced metric, $n^{i}$ is the inward pointing unit vector field normal to $\partial M_{s}, \delta\left(x ; \partial M_{s}\right)$ is the one-sided delta function, and $K_{i k}=h_{i}^{l} h_{k}^{m} \nabla_{l} n_{m}$ is the extrinsic curvature tensor of the boundary.

Let us denote by $\left\{\varphi_{\sigma}^{(+)}(x), \varphi_{\sigma}^{(-)}(x)\right\}$ a complete set of positive and negative energy solutions of the field equation obeying the periodicity condition (4) and the boundary condition (5). The collective index $\sigma$ specifies the set of quantum numbers specifying the solutions. For the background geometry under consideration the mode functions can be taken as

$$
\varphi_{\sigma}^{( \pm)}(x)=z^{D / 2}\left[C_{1} J_{\nu}(\lambda z)+C_{2} Y_{\nu}(\lambda z)\right] \exp \left[i \sum_{l=1}^{D-1} k_{l} x^{l} \mp i \omega t\right],
$$

where $J_{\nu}(\lambda z)$ and $Y_{\nu}(\lambda z)$ are the Bessel and Neumann functions, $\omega^{2}=$ $\lambda^{2}+\sum_{l=1}^{D-1} k_{l}^{2}$, and

$$
\nu=\sqrt{D^{2} / 4-D(D+1) \xi+m^{2} a^{2}}
$$

If the order $\nu$ of the cylinder functions is imaginary the corresponding vacuum state is unstable [?]. For this reason we will consider only the values 
for the parameters in the problem for which $\nu \geq 0$. For the components of the momentum one has $-\infty<k_{l}<+\infty$, for $l=1, \ldots, D-2$, and

$$
k_{D-1}=k(n)=2 \pi \frac{n+\tilde{\alpha}}{L}, n=0, \pm 1, \pm 2, \ldots
$$

The complete set of quantum numbers $\sigma$ consists of the components of momentum $k_{r}, r=1,2, \ldots, D-1$, and the "radial" quantum number $\lambda$.

The nonzero components of the induced metric on the brane $y=y_{0}$ and the corresponding extrinsic curvature tensor are given by $h_{\mu \nu}=g_{\mu \nu}$ and $K_{\mu \nu}=-n^{(j)} g_{\mu \nu} / a$, where $n^{(1)}=1$ and $n^{(2)}=-1$. For Dirichlet boundary conditions, $\beta^{(j)}=0$, the surface energy-momentum tensor vanishes and in the following discussion we will assume that $\beta^{(j)} \neq 0$. By using the boundary condition (5), for the VEV of the surface energy-momentum tensor (6) one obtains $\left\langle 0\left|T_{\mu}^{(\mathrm{s}) \nu}\right| 0\right\rangle=\delta\left(x ; \partial M_{s}\right)\left\langle\tau_{\mu}^{(j) \nu}\right\rangle$, where

$$
\left\langle\tau_{\mu}^{(j) \nu}\right\rangle=-\delta_{\mu}^{\nu} \frac{n^{(j)}}{a}\left(\xi-\frac{2 \xi-1 / 2}{n^{(j)} \beta^{(j)}} a\right)\left\langle\varphi \varphi^{\dagger}\right\rangle_{j}
$$

Hence, the problem is reduced to the evaluation of the VEV of the field squared on the brane $\left\langle\varphi \varphi^{\dagger}\right\rangle_{y=y_{0}}$. The VEV 10 is diagonal and all the non-zero components are equal. For the observer living on the brane the corresponding energy density $\varepsilon_{j}$ (the energy per unit physical volume on the brane) and the stresses $p_{j}$ are given by $\varepsilon_{j}=\left\langle\tau_{0}^{(j) 0}\right\rangle$ and $p_{j}=-\varepsilon_{j}$. These quantities do not depend on the coordinates on the brane and correspond to a source of the cosmological constant type for the observer on the brane.

\section{Regularized VEV of the field squared}

The main problem in 10 is that the expression in the right-hand side diverges and some regularization procedure is required with subsequent renormalization. Here we follow the zeta function regularization technique. The consideration in the regions $y \geq y_{0}$ (R-region) and $y \leq y_{0}$ (L-region) will be presented separately.

\subsection{R-region}

We start with the R-region. From the boundary condition (5) for the mode functions (7) for the coefficient $C_{2}$ one gets

$$
C_{2}=-C_{1} \bar{J}_{\nu}^{(1)}\left(\lambda z_{0}\right) / \bar{Y}_{\nu}^{(1)}\left(\lambda z_{0}\right), z_{0}=a e^{y_{0} / a} .
$$

Here, for a given function $F(x)$ we defined the notation

$$
\bar{F}^{(j)}(x)=A_{j} F(x)+B_{j} x F^{\prime}(x), A_{j}=1+\frac{D}{2} n^{(j)} \frac{\beta^{(j)}}{a}, \quad B_{j}=n^{(j)} \frac{\beta^{(j)}}{a} .
$$


The coefficient $C_{1}$ is determined from the normalization condition in a way similar to that given in Bellucci et al. (2015).

As a result, the VEV of the field squared on the right surface of the brane, after integrating over the angular part of $\mathbf{K}=\left(k_{1}, \ldots, k_{D-2}\right)$, is presented as

$$
\begin{aligned}
\left\langle\varphi \varphi^{\dagger}\right\rangle_{1}= & \frac{2^{4-D} B_{1}^{2} z_{0}^{D} a^{1-D}}{\pi^{D / 2+1} \Gamma(D / 2-1) L} \sum_{n=-\infty}^{+\infty} \int_{0}^{\infty} \mathrm{d} K K^{D-3} \\
& \times \int_{0}^{\infty} \mathrm{d} \lambda \lambda \frac{\left[\bar{J}_{\nu}^{(1) 2}\left(\lambda z_{0}\right)+\bar{Y}_{\nu}^{(1) 2}\left(\lambda z_{0}\right)\right]^{-1}}{\sqrt{\lambda^{2}+K^{2}+k^{2}(n)}} .
\end{aligned}
$$

In this and the following sections we will omit the $y=y_{0}$ subscript to the VEV. The expression in the right-hand side of (13) is divergent and requires the regularization with the further renormalization.

For the regularization we define the function

$$
\begin{aligned}
\Phi_{1}(s)= & \frac{2^{4-D} a^{1-D} z_{0}^{D} B_{1}^{2}}{\pi^{D / 2+1} \Gamma(D / 2-1) \mu^{s+1} L} \sum_{n=-\infty}^{+\infty} \int_{0}^{\infty} \frac{\lambda \mathrm{d} \lambda}{\bar{J}_{\nu}^{(1) 2}\left(\lambda z_{0}\right)+\bar{Y}_{\nu}^{(1) 2}\left(\lambda z_{0}\right)} \\
& \times \int_{0}^{\infty} \frac{K^{D-3} \mathrm{~d} K}{\left[\lambda^{2}+K^{2}+k^{2}(n)\right]^{-s / 2}}
\end{aligned}
$$

where $\mu$ is an arbitrary mass scale introduced to keep the dimensionality of the expression. The computation of the VEV of the field squared requires the analytic continuation of the function $\Phi(s)$ to the value $s=-1:\left\langle\varphi \varphi^{\dagger}\right\rangle_{1}=$ $\left.\Phi_{1}(s)\right|_{s=-1}$. Evaluating the integral over $K$ we obtain

$$
\Phi_{1}(s)=\frac{B_{1} a^{1-D} z_{0}^{D}}{(4 \pi)^{\frac{D-2}{2}} L} \sum_{n=-\infty}^{+\infty} \zeta_{(1) n}(s)
$$

where the generalized partial zeta function is defined as

$$
\zeta_{(1) n}(s)=\frac{2 B_{1} \Gamma\left(-\alpha_{s}\right)}{\pi^{2} \Gamma(-s / 2) \mu^{s+1}} \int_{0}^{\infty} \frac{\lambda\left[\lambda^{2}+k^{2}(n)\right]^{\alpha_{s}} \mathrm{~d} \lambda}{\bar{J}_{\nu}^{(1) 2}\left(\lambda z_{0}\right)+\bar{Y}_{\nu}^{(1) 2}\left(\lambda z_{0}\right)},
$$

where $\alpha_{s}=(D+s) / 2-1$. Presenting the integrand in terms of the Hankel functions and rotating the integration contour in the complex plane $\lambda$, the following integral representation of the zeta function is obtained

$$
\zeta_{(1) n}(s)=-\frac{\mu^{-s-1}}{\Gamma(-s / 2) \Gamma\left(\alpha_{s}+1\right)} \int_{k(n)}^{\infty} \mathrm{d} \lambda \lambda\left[\lambda^{2}-k^{2}(n)\right]^{\alpha_{s}} \frac{K_{\nu}\left(\lambda z_{0}\right)}{\bar{K}_{\nu}^{(1)}\left(\lambda z_{0}\right)},
$$

valid in the slice $-D<\operatorname{Re} s<1-D$ of complex plane $s$. 


\subsection{L-region}

In the L-region, from the regularity condition on the AdS boundary $z=0$ one gets $C_{2}=0$ and the coefficient $C_{1}$ is determined from the normalization condition. From the boundary condition at $y=y_{0}$ it follows that the eigenvalues of $\lambda$ are the roots of the equation $\bar{J}_{\nu}^{(2)}\left(\lambda z_{0}\right)=0$. We will denote these roots by $\lambda=\lambda_{l}, l=1,2, \ldots$ For the corresponding regularized VEV one finds

$$
\Phi_{2}(s)=\frac{B_{2} a^{1-D} z_{0}^{D}}{(4 \pi)^{\frac{D-2}{2}} L} \sum_{n=-\infty}^{+\infty} \zeta_{(2) n}(s)
$$

where the generalized partial zeta function is defined as

$$
\zeta_{(2) n}(s)=-\frac{\Gamma\left(-\alpha_{s}\right)}{\Gamma(-s / 2) \mu^{s+1}} \sum_{l=1}^{\infty} \lambda_{l} \frac{\left[\lambda_{l}^{2}+k^{2}(n)\right]^{\alpha_{s}} J_{\nu}\left(\lambda_{l} z_{0}\right)}{\left[\partial_{\lambda} \bar{J}_{\nu}^{(2)}\left(\lambda z_{0}\right)\right]_{\lambda=\lambda_{l}}} .
$$

Taking into account the fact that $x=\lambda_{l} z_{0}$ is a simple zero of $\bar{J}_{\nu}^{(2)}(x)$ and using the residue theorem we get the representation

$$
\zeta_{(2) n}(s)=-\frac{\Gamma\left(-\alpha_{s}\right)}{2 \pi i \Gamma(-s / 2) \mu^{s+1}} \int_{C} \mathrm{~d} u u\left[u^{2}+k^{2}(n)\right]^{\alpha_{s}} \frac{J_{\nu}\left(u z_{0}\right)}{\bar{J}_{\nu}^{(2)}\left(u z_{0}\right)},
$$

where $C$ is a closed counterclockwise contour in the complex plane $u$, enclosing all the zeros $\lambda_{l}$. Deforming the contour $C$, the following integral representation

$$
\zeta_{(2) n}(s)=\frac{\mu^{-s-1}}{\Gamma\left(\alpha_{s}+1\right) \Gamma(-s / 2)} \int_{k(n)}^{\infty} \mathrm{d} \lambda \lambda\left[\lambda^{2}-k^{2}(n)\right]^{\alpha_{s}} \frac{I_{\nu}\left(\lambda z_{0}\right)}{\bar{I}_{\nu}^{(2)}\left(\lambda z_{0}\right)},
$$

is obtained in the $-D<\operatorname{Re} s<1-D$ slice of complex plane $s$.

We combine the expressions (17) and (21) into a single expression

$$
\zeta_{(j) n}(s)=\frac{-n^{(j)} \mu^{-s-1}}{\Gamma\left(\alpha_{s}+1\right) \Gamma(-s / 2)} \int_{k(n)}^{\infty} \mathrm{d} \lambda \lambda\left[\lambda^{2}-k^{2}(n)\right]^{\alpha_{s}} \frac{F_{\nu}\left(\lambda z_{0}\right)}{\bar{F}_{\nu}^{(j)}\left(\lambda z_{0}\right)} .
$$

In this expression $F=K$ when $j=1$ and $F=I$ when $j=2$. Thus, for the functions $\Phi(s)$ we have

$$
\Phi_{j}(s)=\frac{B_{j} a^{1-D} z_{0}^{D}}{(4 \pi)^{D / 2-1} L} \sum_{n=-\infty}^{+\infty} \zeta_{(j) n}(s) .
$$

This expression is valid in the slice $-D<\operatorname{Re} s<1-D$ and with the condition that $\bar{F}_{\nu}^{(j)}(u)$ does not have real zeros. To obtain the value at the physical point $s=-1$, an analytic continuation is needed. 


\section{Renormalization and the energy density}

For non-zero $B_{j}$ and for large values of $u$, the ratio in the integrand has the following asymptotic expansion

$$
\frac{F_{\nu}(u)}{\bar{F}_{\nu}^{(j)}(u)} \sim \frac{1}{B_{j}} \sum_{l=1}^{\infty} \frac{v_{l}^{(F, j)}}{u^{l}} .
$$

The coefficients for $I_{\nu}(u)$ and $K_{\nu}(u)$ are related as $v_{l}^{(K, j)}=(-1)^{l} v_{l}^{(I, j)}$. The first four coefficients in the series (24) are

$$
\begin{aligned}
& v_{1}^{(I, j)}=1, \quad v_{2}^{(I, j)}=\frac{1}{2}-\frac{A_{j}}{B_{j}}, \quad v_{3}^{(I, j)}=\frac{3}{8}-\frac{A_{j}}{B_{j}}+\frac{A_{j}^{2}}{B_{j}^{2}}-\frac{\nu^{2}}{2}, \\
& v_{4}^{(I, j)}=\frac{3}{8}+\frac{A_{j}}{B_{j}}\left(\nu^{2}-1\right)+\frac{3}{2} \frac{A_{j}^{2}}{B_{j}^{2}}-\frac{A_{j}^{3}}{B_{j}^{3}}-\nu^{2} .
\end{aligned}
$$

For non-zero Kaluza-Klein modes $k(n)$ we subtract and add to the fraction under integral in Eq. (22) the $N$ leading terms of (24) and integrate the added part explicitly

$$
\begin{aligned}
\zeta_{(j) n}(s)=-\frac{n^{(j)} z_{0}^{-2 \alpha_{s}-2}}{\Gamma(-s / 2) \mu^{s+1}}\left\{\int_{k(n) z_{0}}^{\infty} d u\left[u^{2}-k^{2}(n) z_{0}^{2}\right]^{\alpha_{s}} \frac{u G_{j}(u)}{\Gamma\left(\alpha_{s}+1\right)}\right. \\
\left.+\frac{1}{2 B_{j}} \sum_{l=1}^{N} \frac{v_{l}^{(F, j)} \Gamma\left(l / 2-\alpha_{s}-1\right)}{\Gamma(l / 2)\left(k^{2}(n) z_{0}\right)^{l-2 \alpha_{s}-2}}\right\},
\end{aligned}
$$

with the notation

$$
G_{j}(u)=\frac{F_{\nu}(u)}{\bar{F}_{\nu}^{(j)}(u)}-\frac{1}{B_{j}} \sum_{l=1}^{N} \frac{v_{l}^{(F, j)}}{u^{l}} .
$$

If a zero mode $k(n)=0$ exists, then we separate it from the sum in Eq. (23) and divide the corresponding integral into parts over the regions $(0,1)$ and $(1, \infty)$ and apply the same procedure for the integral over the latter interval. As a result the following expression is obtained

$$
\begin{aligned}
\Phi_{j}(s)= & -\frac{n^{(j)} a^{1-D} B_{j} \mu^{-s-1} z_{0}^{D-2-2 \alpha_{s}}}{(4 \pi)^{D / 2-1} \Gamma(-s / 2) \Gamma\left(\alpha_{s}+1\right) L} \\
& \times\left[G_{j}^{(s)}+\sum_{n=-\infty}^{+\infty} \int_{u_{n}}^{\infty} d u u\left[u^{2}-k^{2}(n) z_{0}^{2}\right]^{\alpha_{s}} G_{j}(u)\right. \\
& \left.+\frac{\Gamma\left(\alpha_{s}+1\right)}{2 B_{j}} \sum_{l=1}^{N} \frac{v_{l}^{(F, j)} \Gamma\left(l / 2-\alpha_{s}-1\right) \zeta_{0}\left(l / 2-\alpha_{s}-1\right)}{z_{0}^{l-2 \alpha_{s}-2} \Gamma(l / 2)}\right]
\end{aligned}
$$


where $u_{n}=k(n) z_{0}+\delta_{0 k(n)}$ and the part with

$$
G_{j}^{(s)}=\int_{0}^{1} d u u^{2 \alpha_{s}+1} \frac{F_{\nu}(u)}{\bar{F}_{\nu}^{(j)}(u)}-\frac{1}{B_{j}} \sum_{l=1}^{N} \frac{v_{l}^{(F, j)}}{2 \alpha_{s}+2-l},
$$

comes from the zero mode and should be omitted if the problem at hand does not have a zero mode. The local zeta function $\zeta_{0}(x)$ in 28$)$ is defined as $\zeta_{0}(x)=\sum_{n=-\infty}^{+\infty} 1 / k^{2 x}(n)$, where the prime on the summation sign means that the zero mode $k(n)=0$ is excluded from the sum.

The integral under the second sum on the right of $(28)$ is convergent if $N>D+\operatorname{Re} s-1$, and the sum over $k(n)$ is convergent when $N>D+\operatorname{Re} s$. Thus, if $N \geq D$ the divergencies in (28) at the point $s=-1$ come from the first term in the square brackets (a simple pole contained in the summand $l=D-1)$ and from the term with the local zeta function. In what follows we will restrict ourselves to discussing the case $\tilde{\alpha} \neq 0$. So for the phase $\tilde{\alpha}$ we assume the range $0<\tilde{\alpha}<1$. For the local zeta function one has $\zeta_{0}(x)=(L / 2 \pi)^{2 x} \sum_{n=-\infty}^{+\infty}(n+\tilde{\alpha})^{-2 x}$, and consequently

$$
\zeta_{0}(x)=(L / 2 \pi)^{2 x}\left[\zeta_{H}(2 x, 1-\tilde{\alpha})+\zeta_{H}(2 x, \tilde{\alpha})\right],
$$

where $\zeta_{H}(x ; p)$ is the Hurwitz zeta function. The local zeta function enters into the expression 28$]$ for the VEV in the form

$$
P(x, p)=\Gamma(x)\left[\zeta_{H}(2 x, p)+\zeta_{H}(2 x, 1-p)\right], \quad 0<p<1 .
$$

It can be seen that the function $P(x, p)$ has a single simple pole located at $x=1 / 2$.

For general $N$, we have the following expansion around the point $s=-1$ :

$$
\Phi_{j}(s)=\frac{n^{(j)}(2 a)^{1-D} v_{D}^{(F, j)}}{\Gamma(D / 2)(s+1)}+\left\langle\varphi \varphi^{\dagger}\right\rangle_{\mathrm{f}}+\cdots,
$$

where the dots stand for the terms vanishing for $s=-1$ and for the finite part one has

$$
\begin{aligned}
\left\langle\varphi \varphi^{\dagger}\right\rangle_{j, \mathrm{f}}= & -\frac{2(4 \pi)^{\frac{1-D}{2}} n^{(j)} B_{j} z_{0}}{\Gamma\left(\frac{D-1}{2}\right) a^{D-1} L} \sum_{n=-\infty}^{\infty} \int_{k(n) z_{0}}^{\infty} \mathrm{d} u u\left[u^{2}-z_{0}^{2} k^{2}(n)\right]^{\frac{D-3}{2}} \\
\times G_{j}(u)- & \frac{n^{(j)} a^{1-D}}{2^{D} \sqrt{\pi}}\left[\frac{(2 \pi)^{D}}{\left(L / z_{0}\right)^{D}} \sum_{l=1}^{N} \frac{v_{l}^{(F, j)}\left(L / z_{0}\right)^{l}}{(2 \pi)^{l} \Gamma(l / 2)} P\left(\frac{l-D+1}{2}, \tilde{\alpha}\right)\right. \\
& \left.-\frac{\sqrt{\pi} v_{D}^{(F, j)}}{\Gamma(D / 2)}\left(\psi(\tilde{\alpha})+\psi(1-\tilde{\alpha})+2 \ln \left(\frac{2 \pi}{L \mu}\right)\right)\right] .
\end{aligned}
$$

The prime on the summation sign over $l$ means that the summand corresponding to $l=D$ for which the argument of the Hurwitz zeta functions 
becomes 1 is omitted. The structure of the pole term in (33) allows us to absorb them into the corresponding counterterms. For the finite part of the surface energy density one gets

$$
\varepsilon_{j, \mathrm{f}}=-\frac{n^{(j)}}{a}\left(\xi-\frac{2 \xi-1 / 2}{n^{(j)} \beta^{(j)}} a\right)\left\langle\varphi \varphi^{\dagger}\right\rangle_{j, \mathrm{f}} .
$$

To obtain the total energy density on the brane the contributions coming from the two sides of the brane should be summed. One can see, that for odd $D$ and boundary conditions with $n^{(1)} \beta^{(1)}=n^{(2)} \beta^{(2)}=\beta$ the pole parts in the expression of energy density coming from the two sides of the brane cancel each other and finite expression remains

$$
\begin{aligned}
\varepsilon= & -\frac{1}{a^{D}}\left(\xi-\frac{2 \xi-1 / 2}{\beta} a\right)\left\{\frac{2(4 \pi)^{(1-D) / 2} z_{0} \beta}{\Gamma((D-1) / 2) L a} \sum_{n=-\infty}^{\infty} \int_{k(n) z_{0}}^{\infty} \mathrm{d} u u\right. \\
& \times\left[u^{2}-z_{0}^{2} k^{2}(n)\right]^{\frac{D-3}{2}}\left[\frac{K_{\nu}(u)}{\bar{K}_{\nu}^{(1)}(u)}+\frac{I_{\nu}(u)}{\bar{I}_{\nu}^{(2)}(u)}-\frac{a}{\beta} \sum_{l=1}^{[N / 2]} \frac{2 v_{2 l}^{(I, 2)}}{u^{2 l}}\right] \\
& \left.+\frac{2 \pi^{D-1 / 2}}{\left(L / z_{0}\right)^{D}} \sum_{l=1}^{[N / 2]} \frac{v_{2 l}^{(I, 2)}\left(L / z_{0}\right)^{2 l}}{\Gamma(l)(2 \pi)^{2 l}} P\left(\frac{2 l-D+1}{2}, \tilde{\alpha}\right)\right\} .
\end{aligned}
$$

Here $[N / 2]$ means the integer part of $N / 2$. Note that the expression (35) does not depend on the mass scale $\mu$. The energy density (35) is an even periodic function of the magnetic flux $\Phi$ enclosed by compact dimension with the period equal to the flux quantum $\Phi_{0}$.

The cosmological constant measured in units of the AdS curvature scale $a, a^{D} \varepsilon$, depends on the ratio $\beta / a$, on the parameter $\tilde{\alpha}$, determining the magnetic flux enclosed by the compact dimension and on the ratio $L / z_{0}$. Note that the energy density depends on the length of the compact dimension and on the location of the brane in the form of the combination $L / z_{0}$. The latter property is a consequence of the maximal symmetry of the AdS spacetime.

\section{Conclusion}

We have investigated the VEV of the surface energy-momentum tensor for a charged scalar field, induced on a brane in background of a locally AdS spacetime with a compact spatial dimension. The brane is parallel to the AdS boundary and the field operator obeys Robin boundary condition on it. In general, the Robin coefficients on the left and right hand sides of the brane are different. The VEV of the surface energy-momentum tensor is expressed in terms of the VEV for the field squared. The latter is divergent and a regularization is required with the further renormalization. Here we have used the generalized zeta function regularization scheme. In this way, 


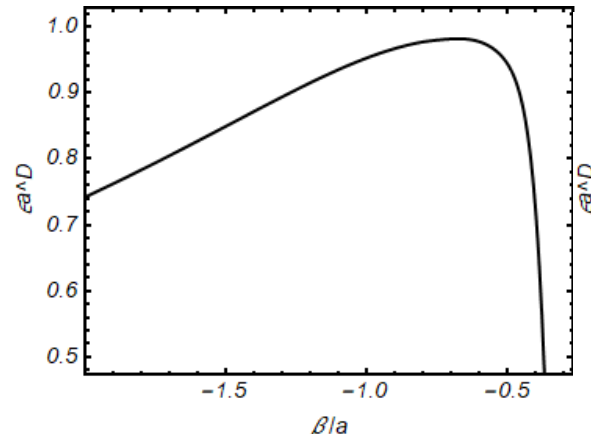

(a)

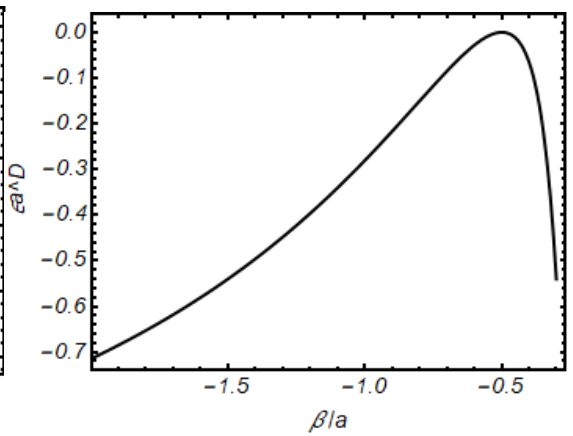

(b)

Figure 1: The dependence of $a^{D} \epsilon$ (determined by the eq. (35p) on the ratio $\beta / a$ with fixed values of $z_{0} / L=1, \quad \tilde{\alpha}=0.45$, for minimally (a) and conformally (b) coupled massless scalar fields $(m=0)$.

for both regions $y \geq y_{0}$ (R-region) and $y \leq y_{0}$ (L-region) the VEV of the field squared on the brane is decomposed into the pole and finite parts. The finite parts depend on the normalization mass scale $\mu$. This dependence disappears in the total energy density on the brane for odd values of the spatial dimension if the Robin coefficients for the R- and L-regions are related as $\beta_{1}=-\beta_{2}$. In this special case, the VEV of the surface energy density is given by (4.17) and for the corresponding pressures one has $p=-\varepsilon$. This corresponds to a cosmological constant induced on the brane by quantum effects.

\section{References}

Aharony, O.; Gubser, S. S.; Maldacena, J.; Ooguri, H.; Oz, Y. 2000, Phys. Rep., 323, 183

Bellucci, S.; Saharian, A. A.; Vardanyan, V. 2015, JHEP, 11, 092

Bellucci, S.; Saharian, A. A.; Vardanyan, V. 2016, Phys. Rev. D, 93, 084011

Breitenlohner, P.; Freedman, D. Z. 1982, Ann. Phys. (NY) 144, 249

Bezerra de Mello, E. R.; Saharian, A. A.; Setare, M. R. 2015, Phys. Rev. D, 92, 104005

Bezerra de Mello, E. R.; Saharian, A. A.; Vardanyan, V. 2015, Phys. Lett. B, 741, 155

Bordag, M.; Klimchitskaya, G. L.; Mohideen, U.; Mostepanenko, V. M. 2009, Advances in the Casimir Effect (Oxford University Press, Oxford, 2009)

Brax, P.; Van de Bruck, C. 2003, Classical Quantum Gravity, 20, 201

Casimir Physics 2001, edited by D. Dalvit, P. Milonni, D. Roberts, 
and F. da Rosa, Lecture Notes in Physics Vol. 834 (Springer-Verlag, Berlin, 2011)

Elizalde, E.; Odintsov, S. D.; Saharian, A. A. 2013, Phys. Rev. D, 87, 084003

Knapman, A.; Toms, D. J. 2004, Phys. Rev. D, 69044023

Maartens, R., K. Koyama, K. 2010, Living Rev. Relativity, 13, 5

Martin, J. 2012, C. R. Physique, 13, 566

Milton, K. A. 2002, The Casimir Effect: Physical Manifestation of Zero-Point Energy (World Scientific, Singapore, 2002)

Saharian, A. A. 2004, Phys. Rev. D, 70, 064026

Saharian, A. A. 2005, Nucl. Phys. B, 712, 196

Saharian, A. A. 2006, Phys. Rev. D 73, 064019

Saharian, A. A. 2004, Phys. Rev. D, 69, 085005

Saharian, A. A. 2004, Phys. Rev. D, 70, 064026

Saharian, A. A. 2006, Phys. Rev. D, 74, 124009

Saharian, A. A.; Sargsyan, H. G. 2018, Astrophysics, 61, 375

Shao, S.-H.; Chen, P.; Gu, J.-A. 2010, Phys. Rev. D, 81, 084036 